\title{
Evaluation of Iranian Students' Mental Health: A Cross-sectional Survey
}

\author{
Meisam Akhlaghdoust ${ }^{*}{ }^{* \mathbb{D}}$, Poorya Davoodi ${ }^{2}$, Atousa Hashemi ${ }^{3}$, Farzan Fathalizade ${ }^{4}$, Matin Baghani ${ }^{2}$, Fatemeh \\ Kamalipoor $^{5}$, Yasaman Rajaee ${ }^{5}$, Sara Mohammadi Doust ${ }^{5}$, Tooba Ebadi Fard Azar ${ }^{5}$, Faezeh Eslami ${ }^{5}$, Farzaneh \\ Ghasemi $^{5}$, Mahdi Akhlaghdoost ${ }^{6}$
}

${ }^{1}$ Functional Neurosurgery Research Center, Shahid Beheshti University of Medical Sciences, Tehran, Iran ${ }^{2}$ Pars Advanced and Minimally Invasive Medical Manners Research Center, Pars Hospital, Iran University of Medical Sciences, Tehran, Iran

${ }^{3}$ Young Researchers and Elite Club, Yadegar-e-Imam Khomeini, Shahr-e-Rey Branch, Islamic Azad University, Tehran, Iran ${ }^{4}$ Minimally Invasive Techniques Research Center in Women, Tehran Medical Sciences Branch, Islamic Azad University, Tehran, Iran

${ }^{5}$ Islamic Azad University, Tehran Medical Sciences Branch, Tehran, Iran

${ }^{6}$ Islamic Azad University, Science and Research Branch, Tehran, Iran

\begin{abstract}
Background: Mental health is a significant problem in developed and developing countries which influences all aspects of life. We aimed to evaluate the status of Iranian students' mental health. Methods: This cross-sectional study was performed on 2052 elementary school students located in Tehran, Iran, in September 2018. We used the Census sampling method for sampling and the general health questionnaire comprising 28 items. For statistical analysis SPSS software, version 21 was used.

Results: A total of 2052 elementary school students (1373 girls and 679 boys) participated in this study. The mean \pm standard deviation $(\mathrm{SD})$ ages of the girls and boys were $14.1 \pm 1.7$ and $13.9 \pm 1.1$ years, respectively. The mean \pm SD of anxiety, social dysfunction, and depression scores were $7.42 \pm 4.92,7.35 \pm 3.33,6.83 \pm 2.26$ in the girls and $5.65 \pm 3.89,6.30 \pm 3.09,4.61 \pm 1.74$ in the boys, respectively. The mean \pm SD total scores in boys and girls were $20.48 \pm 12.31$ and $27.90 \pm 14.66$, respectively.

Conclusion: The mental health status of elementary school students was generally low, especially in male students.
\end{abstract}

Keywords: General health questionnaire; Student; Mental health.

*Correspondence to
Meisam Akhlaghdoust,
Functional Neurosurgery
Research Center, Tehran, Iran.
Tel: +98212272421;
Email: Akhlaghdoust@sbmu.
ac.ir
Published online March 27,
2021 .

*Correspondence to Meisam Akhlaghdoust, Research Center, Tel: +98212272421; Email: Akhlaghdoust@sbmu.

Published online March 27 2021

Citation: Akhlaghdoust M, Davoodi P, Hashemi A, Fathalizade F, Baghani M, Kamalipoor F, et al. Evaluation of Iranian students' menta health: a cross-sectional survey. Clin Neurosci J. 2021;8(2):76-79. doi:10.34172/icnj.2021.16.

\section{Introduction}

Iran has an enormous young population whose psychological health can be directly or indirectly threatened by several stressors since this population is more sensitive towards their surroundings. ${ }^{1,2}$ A stressful situation can lead to catastrophic consequences in young people by changing the nervous, endocrine, and immune systems. ${ }^{3}$ If these problems become chronic, they can lead to illness and dysfunction in adolescents. ${ }^{4}$ Moreover, Having poor general health affects all aspects of young people's lives. ${ }^{4,5}$ Mental health problems can have negative economic and social outcomes, so the early detection of these problems among young people can have significant benefits; however, since there is no clear and precise definition of mental health, this aim is sometimes not easy to reach. ${ }^{5}$

According to the reports of the World Health
Organization (WHO), approximately 450 million people suffer from mental, behavioral, or neurological problems. ${ }^{6-9}$ Besides, it estimated that $20 \%$ of children worldwide have a mental health problem, and $16.3 \%$ of students are prone to mental disorders. ${ }^{10}$ Depressive disorder is the most prevalent mental disorder with a rate of $16 \%-30 \%,{ }^{4,7}$ Based on the results of studies published in recent years, around $30 \%-40 \%$ of youth in the United States have been diagnosed with at least one mental disorder. ${ }^{11}$ However, there is a lake of information about psychological problems in Iran; the outcomes of these studies are important for timely treatment because most young people who are suffering from mental health problems do not seek help and schools are suitable for screening and identifying high-risk people. In this cross-sectional study, we aimed to evaluate mental and psychological problems among Iranian elementary

(C) 2021 The Author(s). This is an open access article distributed under the terms of the Creative Commons Attribution License (http:// creativecommons.org/licenses/by/4.0/), which permits unrestricted use, distribution, and reproduction in any medium, provided the original work is properly cited. 
students using the General Health Questionnaire (GHQ) as a screening tool that is available and valid. It was designed by Goldberg in 1972 for the first time to assess mental well-being and is confirmed by the WHO. We hope that the study provides useful information for future investigations.

\section{Materials and Methods}

\section{Design, Participants, and Procedures}

This cross-sectional survey was conducted on 2052 elementary school students in four schools (two girls' schools and two boys' schools) located in the southeast of Tehran in September 2018. We used the census sampling method. We included 13-15 year-old students, and excluded those with malnutrition and genetic disorders. Demographic data, body mass index (BMI), tobacco experience, alcohol consumption, and history of drug abuse were collected by a checklist. This survey was performed by a clinical psychologist and a medical doctor.

\section{Questionnaire}

The GHQ was created by Goldberg and colleagues and confirmed by the WHO. ${ }^{12}$ It is comprised by 28 items and has four categories. Each category consists of seven items: physical symptoms (items 1-7), anxiety (items 8-14), social dysfunction (items 15-21), and depression (items 22-28). Each item is scored on a four-point Likert scale ( $0=$ not at all, $1=$ usually, $2=$ mostly, $3=$ almost always). The total score ranges from zero to 84 . A cut-off point of 23 was determined in this survey based on Cronbach's alpha coefficient test.

\section{Statistical Analysis}

We used SPSS (SPSS Inc. Chicago, IL, United States) software, version 21 for statistical analysis. $P<0.055$ was considered as statistically significant. Fisher's exact and Chi-square tests were used and correlation coefficients were obtained.

\section{Results}

We enrolled 2052 elementary school students in this study (Table 1). 1373 students were girls, and 679 were men. The mean ages of the girls and boys were 14.1 \pm 1.7 and $13.9 \pm 1.1$ years, respectively. There was a significant difference in alcohol $(P=0.006)$, tobacco $(P=0.003)$, drug $(P=0.001)$ use and BMI $(P=0.049)$ between the girls and boys.

Also, 381 (27.7\%) girls and 493 (72.6\%) boys had experienced smoking cigarette or other types of tobacco. $1.2 \%$ of the girls and $16.1 \%$ of the boys a history of alcohol consumption. $7.3 \%$ of the girls and $13.8 \%$ of the boys had a history of drug usage (Table 1).

As shown in Table 2, a significant difference was seen in physical symptoms, anxiety, social dysfunction, depression, and total scores between female and male
Table 1. Demographic Characteristics of the Students

\begin{tabular}{|c|c|c|c|}
\hline Variables & $\begin{array}{c}\text { Girl } \\
(n=1373) \\
\text { No. }(\%)\end{array}$ & $\begin{array}{c}\text { Boy } \\
(n=679) \\
\text { No. }(\%)\end{array}$ & $P$ Value \\
\hline Age (y) & & & 0.091 \\
\hline 13 & $564(41.1)$ & $320(47.1)$ & \\
\hline 14 & $349(25.4)$ & $175(25.8)$ & \\
\hline 15 & $460(33.5)$ & $184(27.1)$ & \\
\hline Tobacco use & $381(27.7)$ & $493(72.6)$ & 0.003 \\
\hline Alcohol use & $165(1.2)$ & $109(16.1)$ & 0.006 \\
\hline Substance/drug use & $100(7.3)$ & $94(13.8)$ & 0.001 \\
\hline $\mathrm{BMI}\left(\mathrm{kg} / \mathrm{m}^{2}\right)$ & & & 0.049 \\
\hline Underweight $(<18.5)$ & $317(23.1)$ & $248(36.5)$ & \\
\hline Healthy (18.5-24.9) & 535 (39) & $275(40.5)$ & \\
\hline Overweight (25-29.9) & $364(26.5)$ & $113(16.7)$ & \\
\hline Obesity (30-39.9) & $118(8.6)$ & $41(6)$ & \\
\hline Severe Obesity (>40) & $39(2.8)$ & $2(0.3)$ & \\
\hline
\end{tabular}

Table 2. Comparison of the GHQ Subscales Between the Two Sexes

\begin{tabular}{llcc}
\hline & & Mean \pm SD & $P$ value \\
\hline $\begin{array}{l}\text { Physical } \\
\text { Symptoms }\end{array}$ & Female & $6.39 \pm 3.75$ & \\
& Male & $4.21 \pm 3.16$ & $<0.001$ \\
Anxiety & Female & $7.42 \pm 4.92$ & \\
& Male & $5.65 \pm 3.89$ & $<0.001$ \\
Social dysfunction & Female & $7.35 \pm 3.33$ & \\
& Male & $6.30 \pm 3.09$ & $<0.001$ \\
Depression & Female & $6.83 \pm 2.26$ & \\
& Male & $4.61 \pm 1.74$ & $<0.001$ \\
Total & Female & $27.90 \pm 14.66$ & $<0.001$ \\
& Male & $20.48 \pm 12.31$ & \\
\hline
\end{tabular}

groups $(P=0.001)$. The mean \pm standard deviation $(\mathrm{SD})$ scores of the subscales and the total score was significantly higher in female students than male students. The mean \pm SD total scores in boys and girls were $20.48 \pm 12.31$ and $27.90 \pm 14.66$, respectively.

The regression model in Table 3 showed that age, sex, and BMI had a significant relationship with mental health $(P<0.05)$. Also, there was no significant relationship between smoking, alcohol consumption, and substance abuse with mental health.

\section{Discussion}

In this study, 2052 students of both sexes aged 13-15 years were evaluated. The mean \pm SD total GHQ scores for the girls and boys were $27.90 \pm 14.66$ and $20.48 \pm 12.31$, respectively. Therefore, the most prevalent problems were related to the girls. This finding is consistent with a previous study in which the prevalence of psychological symptoms among girls was significantly higher than boys. ${ }^{13}$ According to this study, high GHQ score was 
Table 3. Relationship with Mental Health and Demographic Variables

\begin{tabular}{|c|c|c|c|c|}
\hline \multirow[t]{2}{*}{ Variables } & \multirow{2}{*}{$\begin{array}{c}\beta \\
\text { Coefficient }\end{array}$} & \multirow[t]{2}{*}{$P$ Value } & \multicolumn{2}{|c|}{$\begin{array}{l}95 \% \text { Confidence Interval } \\
\text { for B }\end{array}$} \\
\hline & & & Lower Limit & Upper Limit \\
\hline Age & 0.734 & 0.046 & 0.056 & 0.059 \\
\hline Sex & -0.022 & 0.003 & -0.103 & -0.022 \\
\hline BMI & -0.044 & 0.021 & -0.014 & -0.000 \\
\hline $\begin{array}{l}\text { Smoking (yes=1 } \\
\mathrm{no}=2 \text { ) }\end{array}$ & -0.045 & 0.055 & -0.209 & -0.104 \\
\hline $\begin{array}{l}\text { Alcohol consumption } \\
\text { (yes }=1 \mathrm{no}=2 \text { ) }\end{array}$ & 0.011 & 0.067 & -0.039 & 0.051 \\
\hline $\begin{array}{l}\text { Substance drug } \\
(\text { yes }=1 \text { no=2) }\end{array}$ & -0.017 & 0.099 & -0.111 & 0.0126 \\
\hline
\end{tabular}

prevalent in female students as well as students who had low physical activity. This could be attributed to puberty and problems related to adolescence. Another study showed that $60.2 \%$ of students had mental issues, and mental health distress was more common in girls. ${ }^{14}$ Another study done in the west of Iran reported that $27 \%$ of boys and $46 \%$ of girls had mental health problems. ${ }^{15}$ The findings indicated a high prevalence of psychological symptoms among young people, especially in females. Mohammadi and colleagues reported that the prevalence of anxiety and depression in Iranian female students was $19.01 \%$ and $19.74 \%$, respectively. ${ }^{5}$

The findings of the current study showed that the rates of tobacco use, alcohol consumption, and drug abuse were higher in boys. According to one study, the main reasons leading to tobacco use among young people were having poor relationships with family members and the desire to escape from daily difficulties and problems. Their study showed that students who had a high nicotine dependence level had a higher risk of mental problems. However, they found no difference in relation to low dependence. ${ }^{16}$ According to the results of a prospective cohort study that measured evidence of common mental disorders, which was done using the self-administered GHQ-12, people with significant mental health problems tended to smoke more and the smoking rate was higher in the youngest age group. ${ }^{17}$

During this study, we had several limitations such as the absence of students at school, doing the survey improperly, the existence of certain religious and cultural beliefs among students and their families, and sexual taboos. We suggest doing a similar study and also taking a similar survey in more populated areas with larger sample size. Among students who have higher living standards and comparing them to the results of our study.

\section{Conclusion}

In this study, we evaluated elementary students' mental health using the GH. Our mental health evaluation indexes were physical symptoms, anxiety, social dysfunction, and depression. The students' mental health state was generally low, especially in male students. This study was implemented in order to assess the effect of different variables such as BMI, cigarette smoking, tobacco and alcohol consumption, and drug abuse on mental health.

\section{Conflict of Interest}

The authors declare that they have no conflict of interests.

\section{Acknowledgments}

The authors appreciate the cooperation of Mr. Mohammad Hashemi, staff of the Ministry of Education of Iran.

Authors> Contribution

\section{Authors' Contribution}

Study concept and design: MeA, PD and MaA, drafting of the manuscript MeA, AH, MB and MaA, FE and FF, FG and critical revision of the manuscript: SMD, and FK Statistical Analysis: YR and TEFA. All of the authors have given final approval of the version to be published.

\section{Funding/Support}

None.

\section{Informed Consent}

Written informed was obtained from the participants. All participants gave consent for anonymous publication of their results.

\section{Ethical Statement}

This study was approved by the ethical research committee of Pars advanced and minimally invasive medical manners research center.

\section{References}

1. Sanchez AL, Cornacchio D, Poznanski B, Golik AM, Chou $\mathrm{T}$, Comer JS. The effectiveness of school-based mental health services for elementary-aged children: a meta-analysis. J Am Acad Child Adolesc Psychiatry. 2018;57(3):153-65. doi: 10.1016/j.jaac.2017.11.022.

2. Farahangiz S, Mohebpour F, Salehi A. Assessment of mental health among Iranian medical students: a cross-sectional study. Int J Health Sci (Qassim). 2016;10(1):49-55. doi: 10.12816/0031216.

3. Emami H, Ghazinour M, Rezaeishiraz H, Richter J. Mental health of adolescents in Tehran, Iran. J Adolesc Health. 2007;41(6):571-6. doi: 10.1016/j.jadohealth.2007.06.005.

4. Pojednic R, Peabody S, Carson S, Kennedy M, Bevans K, Phillips EM. The effect of before school physical activity on child development: a study protocol to evaluate the Build Our Kids Success (BOKS) program. Contemp Clin Trials. 2016;49:103-8. doi: 10.1016/j.cct.2016.06.009.

5. Shahraki-Sanavi F, Rakhshani F, Ansari-Moghaddam A, Mohammadi M. The association of mental health with family relationships and high-risk behaviors in female students of South-Eastern Iran. Health Scope. 2020;9(1):e55998. doi: 10.5812/jhealthscope.55998.

6. Wei Y, McGrath PJ, Hayden J, Kutcher S. Measurement properties of tools measuring mental health knowledge: a systematic review. BMC Psychiatry. 2016;16(1):297. doi: 10.1186/s12888-016-1012-5.

7. Ahmadi K, Saghafi A. Psychosocial profile of Iranian 
adolescents' Internet addiction. Cyberpsychol Behav Soc Netw. 2013;16(7):543-8. doi: 10.1089/cyber.2012.0237.

8. Alimohammadzadeh $\mathrm{K}$, Akhlaghdoust $M$, Bahrainian SA, Mirzaei A, Asadi HK, Jamalpour MR, et al. Survey on Mental Health of Iranian Medical Students: A Cross-sectional Study in Islamic Azad University. Health (Irvine Calif). 2017;62:6-7.

9. Levecque K, Anseel F, De Beuckelaer A, Van der Heyden J, Gisle L. Work organization and mental health problems in PhD students. Res Policy. 2017;46(4):868-79. doi: 10.1016/j. respol.2017.02.008.

10. von der Embse NP, Pendergast LL, Kilgus SP, Eklund KR. Evaluating the applied use of a mental health screener: structural validity of the Social, Academic, and Emotional Behavior Risk Screener. Psychol Assess. 2016;28(10):126575. doi: 10.1037/pas0000253.

11. Macaskill A. Undergraduate mental health issues: the challenge of the second year of study. J Ment Health. 2018;27(3):214-21. doi: 10.1080/09638237.2018.1437611.

12. Goldberg DP, Hillier VF. A scaled version of the General Health Questionnaire. Psychol Med. 1979;9(1):139-45. doi:
$10.1017 / \mathrm{s} 0033291700021644$.

13. Soltanian AR, Nabipour I, Akhondzadeh S, Moeini B, Bahreini F, Barati M, et al. Association between physical activity and mental health among high-school adolescents in Boushehr province: a population based study. Iran J Psychiatry. 2011;6(3):112-6.

14. Sadeghian E, Moghadari Kosha M, Gorji S. The study of mental health status in high school female students in Hamadan city. Avicenna J Clin Med. 2010;17(3):39-45. [Persian].

15. Haghighi A, Dinarvandi A, Afshari P. General mental health status among adolescents in Iran. In: Proceeding of 17th World Congress of the International Association for Child and Adolescent Psychiatry and Allied Professions; 2006. p. 10-4.

16. Üner S, Özcebe H, Telatar TG, Tezcan S. Assessment of mental health of university students with GHQ-12. Turk J Med Sci. 2008;38(5):437-46.

17. Sohrabi MR, Karimi HR, Malih N, Keramatinia AA. Mental health status of medical students in Tehran: a cross sectional study. Soc Determinants Health Soc Determ Health. 2015;1(2):81-8. doi: 10.22037/sdh.v1i2.10348. 